\title{
COMBINATORIAL REES-SUSHKEVICH VARIETIES THAT ARE CROSS, FINITELY GENERATED, OR SMALL
}

\author{
EDMOND W. H. LEE
}

(Received 25 February 2009)

\begin{abstract}
A variety is said to be a Rees-Sushkevich variety if it is contained in a periodic variety generated by 0 -simple semigroups. Recently, all combinatorial Rees-Sushkevich varieties have been shown to be finitely based. The present paper continues the investigation of these varieties by describing those that are Cross, finitely generated, or small. It is shown that within the lattice of combinatorial Rees-Sushkevich varieties, the set $\mathcal{F}$ of finitely generated varieties constitutes an incomplete sublattice and the set $\mathcal{S}$ of small varieties constitutes a strict incomplete sublattice of $\mathcal{F}$. Consequently, a combinatorial ReesSushkevich variety is small if and only if it is Cross. An algorithm is also presented that decides if an arbitrarily given finite set $\Sigma$ of identities defines, within the largest combinatorial Rees-Sushkevich variety, a subvariety that is finitely generated or small. This algorithm has complexity $\mathcal{O}(n k)$ where $n$ is the number of identities in $\Sigma$ and $k$ is the length of the longest word in $\Sigma$.
\end{abstract}

2000 Mathematics subject classification: primary 20M07; secondary 03C05, $08 \mathrm{~B} 15$.

Keywords and phrases: semigroups, 0 -simple, varieties, Rees-Sushkevich, Cross, finitely based, finitely generated, small.

\section{Introduction}

Recall that a semigroup is 0 -simple if it does not contain any nontrivial proper ideals. The class of 0-simple semigroups was one of the first classes of semigroups to be studied, in the pioneering work of Rees [20] and Sushkevich [23], and remains one of the most important and interesting classes of semigroups. Following Kublanovsky $[6,7]$, any subvariety of a periodic variety generated by 0 -simple semigroups is referred to as a Rees-Sushkevich variety. One of the most important results concerning Rees-Sushkevich varieties, due to Mashevitzky [18] and Hall et al. [5], is that for each integer $n \geq 1$, the variety generated by all 0 -simple semigroups over groups of exponent dividing $n$ is finitely based. This positive result, however, does not apply to every Rees-Sushkevich variety as nonfinitely based examples exist in abundance (see, for example, $[2,13,15,17,19])$.

A variety of semigroups is combinatorial if all groups in it are trivial. It follows from results of Hall et al. [5] and Trahtman [25] that the largest combinatorial

(C) 2009 Australian Mathematical Publishing Association Inc. 0004-9727/2009 \$16.00 
Rees-Sushkevich variety coincides with the variety $\mathbf{A}_{2}$ generated by the 0-simple semigroup

$$
A_{2}=\left\langle a, b \mid a^{2}=a b a=a, b^{2}=0, b a b=b\right\rangle
$$

of order 5, and that the variety $\mathbf{A}_{\mathbf{2}}$ is defined by the identities

$$
x^{3} \approx x^{2}, \quad x y x y x \approx x y x, \quad x y x z x \approx x z x y x .
$$

The study of combinatorial Rees-Sushkevich varieties is thus precisely the study of subvarieties of $\mathbf{A}_{\mathbf{2}}$. These varieties have recently been investigated by Reilly, Volkov, and the author (see, for example, [10, 11, 14, 21, 28]). Unlike the general case in which many Rees-Sushkevich varieties containing nontrivial groups are nonfinitely based, all subvarieties of $\mathbf{A}_{\mathbf{2}}$ are finitely based [11]. The lattice $\mathcal{L}\left(\mathbf{A}_{\mathbf{2}}\right)$ of subvarieties of $\mathbf{A}_{\mathbf{2}}$, on the other hand, has a rather complicated structure, for it follows from a result of Vernikov and Volkov [26] that every finite lattice is embeddable in it.

Recall that a variety is small if it contains finitely many subvarieties. The present paper is a continuation of $[11,14]$ in the study of subvarieties of $\mathbf{A}_{2}$ with the objective of investigating those that are finitely generated or small.

\section{THEOREM A.}

(i) Every small subvariety of $\mathbf{A}_{2}$ is finitely generated but not vice versa.

(ii) The finitely generated subvarieties of $\mathbf{A}_{2}$ constitute an incomplete sublattice of $\mathcal{L}\left(\mathbf{A}_{2}\right)$.

(iii) The small subvarieties of $\mathbf{A}_{2}$ constitute an incomplete sublattice of $\mathcal{L}\left(\mathbf{A}_{\mathbf{2}}\right)$.

Since all subvarieties of $\mathbf{A}_{\mathbf{2}}$ are finitely based [11], any subvariety of $\mathbf{A}_{\mathbf{2}}$ is defined within $\mathbf{A}_{\mathbf{2}}$ by some finite set of identities. Therefore, it is reasonable to ask whether or not it is decidable if an arbitrarily given finite set of identities defines a subvariety of $\mathbf{A}_{\mathbf{2}}$ that is finitely generated or small. It turns out that this question has an affirmative answer.

THEOREM B. Suppose that $\Sigma$ is any finite set of $n$ identities formed by words of length at most $k$. Then there exists an algorithm with complexity $\mathcal{O}(n k)$ that decides if the subvariety of $\mathbf{A}_{2}$ defined by $\Sigma$ is finitely generated or small.

In the next section, a partition of the proper subvarieties of $\mathbf{A}_{2}$ into five pairwise disjoint intervals is given. Each of these intervals is then individually investigated with the aim of establishing Theorems A and B. Further details on the organization of this paper are given after Lemma 2.3.

REMARK 1. It follows from [12] that Theorem A(ii) also holds for Rees-Sushkevich varieties of central simple semigroups, that is, the set of finitely generated varieties of central simple semigroups constitutes an incomplete lattice. However, Theorem A does not hold for varieties of semigroups in general since there exists a small variety that is nonfinitely generated (see [22]), the meet of two finitely generated varieties need not be finitely generated (Corollary 3.4(ii)), and the join of two small varieties need not be small [22]. 
REMARK 2. Recall that a variety is Cross if it is finitely based, finitely generated, and small. Since all subvarieties of $\mathbf{A}_{\mathbf{2}}$ are finitely based [11], it follows from Theorem A(i) that a subvariety of $\mathbf{A}_{\mathbf{2}}$ is small if and only if it is Cross. Consequently, any result on small subvarieties of $\mathbf{A}_{\mathbf{2}}$ is also a result on Cross subvarieties of $\mathbf{\mathbf { A } _ { 2 }}$.

\section{Preliminaries}

In this paper, let $\mathcal{X}$ be a countably infinite alphabet. Elements of $\mathcal{X}$ are referred to as letters and elements of the free monoid over $\mathcal{X}$ are referred to as words. The content of a word $\mathbf{w}$, denoted by $\mathrm{C}(\mathbf{w})$, is the set of letters occurring in $\mathbf{w}$.

An identity is typically written as $\mathbf{u} \approx \mathbf{v}$ where $\mathbf{u}$ and $\mathbf{v}$ are words. Let $\Sigma$ be any set of identities. The variety defined by $\Sigma$ is the class of all semigroups that satisfy all identities in $\Sigma$. If $\mathbf{V}$ is a variety, then the subvariety of $\mathbf{V}$ defined by $\Sigma$ is denoted by $\mathbf{V} \Sigma$, and the lattice of subvarieties of $\mathbf{V}$ is denoted by $\mathcal{L}(\mathbf{V})$. Refer to [3, 27] for other undefined notation and terminology in this paper.

The semigroup $A_{2}$ and the combinatorial Brandt semigroup

$$
B_{2}=\left\langle c, d \mid c^{2}=d^{2}=0, c d c=c, d c d=d\right\rangle
$$

of order 5 are, up to isomorphism, the only minimal 0 -simple semigroups with zero divisors. It is routine to verify that the sets

$$
A_{0}=\{0, b, a b, b a\} \quad \text { and } \quad B_{0}=\{0, d, c d, d c\}
$$

are subsemigroups of $A_{2}$ and $B_{2}$, respectively. Denote by $\mathbf{A}_{\mathbf{0}}, \mathbf{B}_{\mathbf{0}}$, and $\mathbf{B}_{\mathbf{2}}$ the varieties generated by the semigroups $A_{0}, B_{0}$, and $B_{2}$, respectively. The variety $\mathbf{A}_{\mathbf{0}}$ is clearly contained in $\mathbf{A}_{2}$. The varieties $\mathbf{B}_{\mathbf{0}}$ and $\mathbf{B}_{2}$ are also contained in $\mathbf{A}_{\mathbf{2}}$ since $\mathbf{A}_{\mathbf{2}}$ is the largest combinatorial Rees-Sushkevich variety.

Lemma 2.1. [11, Proposition 2.5 and Lemma 2.6]

(i) For any variety $\mathbf{V}$ in $\left\{\mathbf{A}_{\mathbf{2}}, \mathbf{B}_{\mathbf{2}}, \mathbf{A}_{\mathbf{0}}, \mathbf{B}_{\mathbf{0}}\right\}$, the subvariety $\overline{\mathbf{V}}$ of $\mathbf{A}_{\mathbf{2}}$ that is largest with respect to not containing $\mathbf{V}$ exists and is finitely based. More specifically,

$$
\begin{aligned}
& \overline{\mathbf{A}}_{\mathbf{2}}=\mathbf{A}_{\mathbf{2}}\left\{x^{2} y^{2} x^{2} \approx x^{2} y x^{2}\right\}, \\
& \overline{\mathbf{B}}_{\mathbf{2}}=\mathbf{A}_{\mathbf{2}}\left\{x y^{2} x \approx x y x\right\}, \\
& \overline{\mathbf{A}}_{\mathbf{0}}=\mathbf{A}_{\mathbf{2}}\left\{x^{2} y^{2} x^{2} y^{2} \approx x^{2} y^{2}\right\}, \\
& \overline{\mathbf{B}}_{\mathbf{0}}=\mathbf{A}_{\mathbf{2}}\left\{x^{2} y^{2} z^{2} \approx x^{2} y z^{2}\right\} .
\end{aligned}
$$

(ii) The inclusions $\overline{\mathbf{B}}_{\mathbf{0}} \subset \overline{\mathbf{A}}_{\mathbf{0}} \subset \overline{\mathbf{A}}_{\mathbf{2}}$ and $\overline{\mathbf{B}}_{\mathbf{0}} \subset \overline{\mathbf{B}}_{\mathbf{2}} \subset \overline{\mathbf{A}}_{\mathbf{2}}$ hold.

Note that the variety $\overline{\mathbf{A}}_{\mathbf{2}}$ is the unique maximal subvariety of $\mathbf{A}_{\mathbf{2}}$ since it contains every proper subvariety of $\mathbf{A}_{\mathbf{2}}$.

LEMMA 2.2.

(i) The varieties $\overline{\mathbf{B}}_{\mathbf{0}}$ and $\overline{\mathbf{B}}_{\mathbf{2}}$ satisfy the identities

$$
x^{2} y^{k} x \approx x y^{k} x \approx x y^{k} x^{2}, \quad x h^{m} y^{2} t^{n} x \approx x h^{m} y t^{n} x,
$$

where $k, m, n \in\{0,1\}$. 
(ii) Every proper subvariety of $\mathbf{A}_{2}$ satisfies the identities

$$
x h^{m} y^{2} z^{2} t^{n} x \approx x h^{m} z^{2} y^{2} t^{n} x,
$$

where $m, n \in\{0,1\}$.

Proof. (i) By [11, Lemma 3.3], the variety $\overline{\mathbf{B}}_{2}$ satisfies the identities (2). By Lemma 2.1(ii), the variety $\overline{\mathbf{B}}_{\mathbf{0}}$ is contained in $\overline{\mathbf{B}}_{\mathbf{2}}$ and so also satisfies the identities (2).

(ii) This follows from [11, Lemma 3.11].

Lemma 2.3. [11, Propositions 2.7] The lattice $\mathcal{L}\left(\overline{\mathbf{A}}_{\mathbf{2}}\right)$ is the disjoint union of the intervals

$$
\begin{aligned}
& \mathcal{I}_{1}=\left[\mathbf{A}_{\mathbf{0}} \vee \mathbf{B}_{2}, \overline{\mathbf{A}}_{2}\right], \\
& \mathcal{I}_{2}=\left[\mathbf{A}_{\mathbf{0}}, \overline{\mathbf{B}}_{2}\right], \\
& \mathcal{I}_{3}=\left[\mathbf{B}_{\mathbf{2}}, \overline{\mathbf{A}}_{\mathbf{0}}\right], \\
& \mathcal{I}_{4}=\left[\mathbf{B}_{\mathbf{0}}, \overline{\mathbf{A}}_{\mathbf{0}} \cap \overline{\mathbf{B}}_{2}\right], \\
& \mathcal{I}_{5}=\mathcal{L}\left(\overline{\mathbf{B}}_{\mathbf{0}}\right) .
\end{aligned}
$$

Section 3 presents an equivalent condition on a set $\Sigma$ of identities for which the variety $\overline{\mathbf{B}}_{\mathbf{0}} \Sigma$ in the interval $\mathcal{I}_{5}$ is finitely generated or small. Section 4 introduces some identities and varieties that are necessary in Section 5 for the complete descriptions of the intervals $\mathcal{I}_{1}, \mathcal{I}_{2}, \mathcal{I}_{3}, \mathcal{I}_{4}$, and

$$
\left[\mathbf{B}_{\mathbf{0}}, \mathbf{A}_{2}\right]=\left\{\mathbf{A}_{2}\right\} \cup \mathcal{I}_{1} \cup \mathcal{I}_{2} \cup \mathcal{I}_{3} \cup \mathcal{I}_{4} .
$$

Section 6 addresses the word problems of three subvarieties of $\mathbf{A}_{\mathbf{2}}$ that are crucial to the algorithm in Theorem B and the determination of its complexity. Based on results from Sections 3-6, the main results stated in the Introduction are proved in Section 7.

\section{The interval $\mathcal{I}_{5}$}

A letter $x$ is simple in a word $\mathbf{w}$ if $x$ occurs exactly once in $\mathbf{w}$. A nonempty word is simple if each of its letters is simple in it. A permutation identity is an identity $\mathbf{u} \approx \mathbf{v}$ where $\mathbf{u}$ and $\mathbf{v}$ are distinct simple words with $\mathrm{C}(\mathbf{u})=\mathrm{C}(\mathbf{v})$. A nontrivial nonpermutation identity $\mathbf{u} \approx \mathbf{v}$ where either $\mathbf{u}$ or $\mathbf{v}$ is simple is said to be diverse.

LEMMA 3.1.

(i) Suppose that some diverse identity is a consequence of some set $\Sigma$ of nontrivial identities. Then $\Sigma$ itself contains some diverse identity.

(ii) An identity is nondiverse if and only if it is a consequence of the identities

$$
x y x \approx z^{2}, \quad x y \approx y x .
$$

PROOF. (i) Suppose that every identity in $\Sigma$ is nondiverse. Then $\Sigma$ can only contain permutation identities and identities formed by nonsimple words. It is then easy to show that no diverse identity can be a consequence of $\Sigma$.

(ii) Since the identities (4) are nondiverse, it follows from part (i) that any consequence of (4) is a nondiverse identity. Conversely, let $\mathbf{u} \approx \mathbf{v}$ be any nondiverse 
identity. It is clear that any permutation identity is a consequence of the identities (4). Therefore, it suffices to assume that both $\mathbf{u}$ and $\mathbf{v}$ are nonsimple words, whence $\mathbf{u}=\mathbf{w}_{1} x \mathbf{w}_{2} x \mathbf{w}_{3}$ and $\mathbf{v}=\mathbf{w}_{4} y \mathbf{w}_{5} y \mathbf{w}_{6}$ for some letters $x, y \in \mathcal{X}$ and some possibly empty words $\mathbf{w}_{1}, \ldots, \mathbf{w}_{6}$. Since

$(4)$

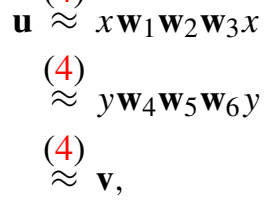

the identity $\mathbf{u} \approx \mathbf{v}$ is a consequence of the identities (4).

LEMMA 3.2. Suppose that $\mathbf{V}$ is any locally finite variety such that the lattice $\mathcal{L}(\mathbf{V})$ satisfies the ascending chain condition. Then $\mathbf{V}$ is a finitely generated variety.

PROOF. For each $n \geq 1$, let $\mathbf{V}_{n}$ be the variety generated by the $\mathbf{V}$-free semigroup over $n$ letters. The varieties in the chain $\mathbf{V}_{1} \subseteq \mathbf{V}_{2} \subseteq \cdots$ are finitely generated because $\mathbf{V}$ is locally finite. Since $\mathcal{L}(\mathbf{V})$ satisfies the ascending chain condition, there exists some $m \geq 1$ such that $\mathbf{V}_{n}=\mathbf{V}_{m}$ for all $n>m$. Consequently, $\mathbf{V}=\bigcup_{n \geq 1} \mathbf{V}_{n}=$ $\mathbf{V}_{m}$ is finitely generated.

PROPOSITION 3.3. The following statements on a set $\Sigma$ of identities are equivalent:

(a) the variety $\overline{\mathbf{B}}_{\mathbf{0}} \Sigma$ is small;

(b) the variety $\overline{\mathbf{B}}_{\mathbf{0}} \Sigma$ is finitely generated;

(c) the set $\Sigma$ contains some diverse identity.

PRoOF. Recall from Lemma 2.1(i) that the variety $\overline{\mathbf{B}}_{\mathbf{0}}$ is defined by the identities (1) and

$$
x^{2} y^{2} z^{2} \approx x^{2} y z^{2}
$$

(a) implies (b). Since $\mathbf{A}_{\mathbf{2}}$ is a finitely generated variety, all its subvarieties are locally finite. Therefore, if the variety $\overline{\mathbf{B}}_{\mathbf{0}} \Sigma$ is small, then it is also finitely generated by Lemma 3.2.

(b) implies (c). Suppose that the variety $\overline{\mathbf{B}}_{\mathbf{0}} \Sigma$ is generated by a finite semigroup $S$ of order $n$. Let $x_{1}, \ldots, x_{n}, y, z_{1}, \ldots, z_{n} \in S$. By [1, Proposition 3.7.1], there exist $s_{1}, s_{2}, s_{3}, t_{1}, t_{2}, t_{3} \in S$ such that $x_{1} \cdots x_{n}=s_{1} s_{2}^{2} s_{3}$ and $z_{1} \cdots z_{n}=t_{1} t_{2}^{2} t_{3}$. Since

$$
\begin{aligned}
x_{1} \cdots x_{n} y z_{1} \cdots z_{n} & =s_{1}\left(s_{2}^{2} s_{3} y t_{1} t_{2}^{2}\right) t_{3} \\
& \stackrel{(5)}{=} s_{1} s_{2}^{2}\left(s_{3} y t_{1}\right)^{2} t_{2}^{2} t_{3} \\
& \stackrel{(2)}{=} s_{1} s_{2}^{2}\left(s_{3} y^{2} t_{1}\right)^{2} t_{2}^{2} t_{3} \\
& \stackrel{(5)}{=} s_{1} s_{2}^{2} s_{3} y^{2} t_{1} t_{2}^{2} t_{3} \\
& =x_{1} \cdots x_{n} y^{2} z_{1} \cdots z_{n},
\end{aligned}
$$


the diverse identity $x_{1} \cdots x_{n} y z_{1} \cdots z_{n} \approx x_{1} \cdots x_{n} y^{2} z_{1} \cdots z_{n}$ is satisfied by the semigroup $S$ and so is a consequence of the identities $\{(1),(5)\} \cup \Sigma$ that define the variety $\overline{\mathbf{B}}_{\mathbf{0}} \Sigma$. It follows from Lemma 3.1(i) that some identity in $\{(1),(5)\} \cup \Sigma$ is diverse. Since every identity in $\{(1),(5)\}$ is nondiverse, $\Sigma$ must contain some diverse identity.

(c) implies (a). Suppose that $\Sigma$ contains some diverse identity

$$
\delta: \mathbf{u} \approx \mathbf{v}
$$

By [16], any variety that satisfies some diverse identity and some permutation identity is small. Therefore, in order to show that the variety $\overline{\mathbf{B}}_{\mathbf{0}} \Sigma$ is small, it suffices to show that it satisfies some permutation identity. By symmetry, there are two cases to consider.

CASE 1. $\mathbf{u}$ is simple and $\mathbf{v}$ is nonsimple. Then $\mathbf{v}=\mathbf{v}_{0} \prod_{i=1}^{n}\left(y \mathbf{v}_{i}\right)$ for some possibly empty words $\mathbf{v}_{0}, \ldots, \mathbf{v}_{n}$ with $n \geq 2$ and some letter $y$ from $\mathcal{X} \backslash \mathrm{C}\left(\mathbf{v}_{0} \cdots \mathbf{v}_{n}\right)$.

(1.1) $y \in \mathrm{C}(\mathbf{u})$. Choose any letters $x_{1}, x_{2}, x_{3}, x_{4} \in \mathcal{X} \backslash \mathrm{C}(\mathbf{u v})$. Denote by $\varphi$ and $\chi$ the substitutions $y \mapsto x_{1} x_{2} x_{3} x_{4}$ and $y \mapsto x_{1} x_{3} x_{2} x_{4}$, respectively. Then $\mathbf{u} \varphi \approx \mathbf{u} \chi$ is clearly a permutation identity. Since

$$
\begin{aligned}
\mathbf{u} \varphi \stackrel{\delta}{\approx} \mathbf{v} \varphi & =\mathbf{v}_{0} \prod_{i=1}^{n}\left(\left(x_{1} x_{2} x_{3} x_{4}\right) \mathbf{v}_{i}\right) \\
& \stackrel{(2)}{\approx} \mathbf{v}_{0} \prod_{i=1}^{n}\left(\left(x_{1} x_{2}^{2} x_{3}^{2} x_{4}\right) \mathbf{v}_{i}\right) \\
& \stackrel{(3)}{\approx} \mathbf{v}_{0} \prod_{i=1}^{n}\left(\left(x_{1} x_{3}^{2} x_{2}^{2} x_{4}\right) \mathbf{v}_{i}\right) \\
& \stackrel{(2)}{\approx} \mathbf{v}_{0} \prod_{i=1}^{n}\left(\left(x_{1} x_{3} x_{2} x_{4}\right) \mathbf{v}_{i}\right) \\
& =\mathbf{v} \chi \stackrel{\delta}{\approx} \mathbf{u} \chi,
\end{aligned}
$$

the variety $\overline{\mathbf{B}}_{\mathbf{0}} \Sigma$ satisfies the identity $\mathbf{u} \varphi \approx \mathbf{u} \chi$.

(1.2) $y \notin \mathrm{C}(\mathbf{u})$. Then $\mathbf{u} y \approx \mathbf{v} y$ is a diverse identity where $\mathbf{u} y$ is simple and $\mathbf{v} y$ is nonsimple. Repeat the argument in Case 1.1 on $\mathbf{u} y \approx \mathbf{v} y$ to obtain a permutation identity that is satisfied by the variety $\overline{\mathbf{B}}_{\mathbf{0}} \Sigma$.

CASE 2. $\mathbf{u}$ and $\mathbf{v}$ are simple. Without loss of generality, assume that $x \in \mathrm{C}(\mathbf{v}) \backslash \mathrm{C}(\mathbf{u})$. Then $\mathbf{u} x \approx \mathbf{v} x$ is a diverse identity where $\mathbf{u} x$ is simple and $\mathbf{v} x$ is nonsimple. Repeat the argument in Case 1 on $\mathbf{u} x \approx \mathbf{v} x$ to obtain a permutation identity that is satisfied by the variety $\overline{\mathbf{B}}_{\mathbf{0}} \Sigma$.

Let $\mathbf{M}$ be the variety generated by the monoid $\left\langle a: a^{3}=a^{2}\right\rangle \cup\{1\}$ and $\mathbf{N}$ be the variety defined by the identities (4). 
COROLlary 3.4.

(i) The set of nonfinitely generated subvarieties of $\overline{\mathbf{B}}_{\mathbf{0}}$ constitutes the interval $\left[\mathbf{N}, \overline{\mathbf{B}}_{\mathbf{0}}\right]$. In particular, the variety $\overline{\mathbf{B}}_{\mathbf{0}}$ is nonfinitely generated.

(ii) The variety $\mathbf{A}_{\mathbf{2}} \cap \mathbf{M}$ is nonfinitely generated.

Proof. (i) It is easy to see that $\mathbf{N}$ is a subvariety of $\overline{\mathbf{B}}_{\mathbf{0}}$. Let $\mathbf{V}$ be a subvariety of $\overline{\mathbf{B}}_{\mathbf{0}}$ so that $\mathbf{V}=\overline{\mathbf{B}}_{\mathbf{0}} \Sigma$ for some set $\Sigma$ of identities. Consider the following conditions:

(a) $\mathbf{N} \subseteq \mathbf{V}$, that is, every identity in $\Sigma$ is a consequence of (4);

(b) every identity in $\Sigma$ is nondiverse;

(c) $\mathbf{V}$ is nonfinitely generated.

Conditions (a) and (b) are equivalent by Lemma 3.1(ii), and conditions (b) and (c) are equivalent by Proposition 3.3.

(ii) It is well known and easy to show that the variety $\mathbf{M}$ is defined by the identities $x^{3} \approx x^{2}$ and $x y \approx y x$ (see, for example, [1, Corollary 6.1.5]). It is then routine to verify that $\mathbf{A}_{\mathbf{2}} \cap \mathbf{M}=\overline{\mathbf{B}}_{\mathbf{0}}\{x y \approx y x\}$; this variety is nonfinitely generated by Proposition 3.3.

\section{The identities $\langle n|$ and $|n\rangle$}

Let $\omega$ be the least infinite ordinal and $\widehat{\omega}=\omega+1$ be its successor. For any $n$ from

$$
\{0,1,2, \ldots, \omega, \widehat{\omega}\}
$$

define the identities

$$
\langle n|: \mathbf{h}_{(n)} x^{2} y^{2} x^{2} \approx \mathbf{h}_{(n)} y^{2} x^{2} y^{2} x^{2} \quad \text { and } \quad|n\rangle: x^{2} y^{2} x^{2} \mathbf{t}_{(n)} \approx x^{2} y^{2} x^{2} y^{2} \mathbf{t}_{(n)}
$$

where

$$
\mathbf{h}_{(n)}=\left\{\begin{array}{ll}
h_{1} \cdots h_{n} & \text { if } 0 \leq n<\omega, \\
h_{1}^{2} h_{2} & \text { if } n=\omega, \\
h^{2} & \text { if } n=\widehat{\omega},
\end{array} \quad \text { and } \quad \mathbf{t}_{(n)}= \begin{cases}t_{1} \cdots t_{n} & \text { if } 0 \leq n<\omega, \\
t_{1} t_{2}^{2} & \text { if } n=\omega, \\
t^{2} & \text { if } n=\widehat{\omega} .\end{cases}\right.
$$

Note that the words $\mathbf{h}_{(0)}$ and $\mathbf{t}_{(0)}$ are empty.

Lemma 4.1. Suppose that $m \leq n \leq \widehat{\omega}$. Then

(i) the identity $\langle n|$ is a consequence of the identities $\{(1),\langle m|\}$;

(ii) the identity $|n\rangle$ is a consequence of the identities $\{(1),|m\rangle\}$.

PROOF. This is straightforward.

LEMMA 4.2. Let $S$ be any finite semigroup of order $n$. Then

(i) $S \vDash\langle\omega|$ implies that $S \vDash\langle n|$;

(ii) $S \vDash|\omega\rangle$ implies that $S \vDash|n\rangle$. 
Proof. Suppose that $S \vDash\langle\omega|$. Let $h_{1}, \ldots, h_{n}, x, y \in S$. By [1, Proposition 3.7.1], there exist $s_{1}, s_{2}, s_{3} \in S$ such that $h_{1} \cdots h_{n}=s_{1} s_{2}^{2} s_{3}$. Then $S \vDash\langle n|$ because

$$
\begin{aligned}
h_{1} \cdots h_{n} x^{2} y^{2} x^{2} & =s_{1} s_{2}^{2} s_{3} x^{2} y^{2} x^{2} \\
& \stackrel{\langle\omega|}{=} s_{1} s_{2}^{2} s_{3} y^{2} x^{2} y^{2} x^{2} \\
& =h_{1} \cdots h_{n} y^{2} x^{2} y^{2} x^{2} .
\end{aligned}
$$

Therefore, part (i) holds. Part (ii) follows by symmetry.

Let $\mathbf{L}_{0}$ be the variety of trivial semigroups and for each $n \in\{1,2, \ldots\}$, let $\mathbf{L}_{n}$ be the subvariety of $\mathbf{A}_{\mathbf{2}}$ defined by the identity

$$
\lambda_{n}: x_{1} \cdots x_{n} y \approx x_{1} \cdots x_{n} .
$$

It is easy to show that the variety $\mathbf{L}_{n}$ does not contain the semigroup $B_{0}$ and so belongs to the interval $\mathcal{I}_{5}$. The identity $\lambda_{n}$ is diverse so that by Proposition 3.3, the variety $\mathbf{L}_{n}$ is both finitely generated and small. For each $n<\omega$, let $\mathbf{R}_{n}$ be the variety that is the symmetrical dual of $\mathbf{L}_{n}$. Similarly, $\mathbf{R}_{n}$ is a finitely generated and small variety in the interval $\mathcal{I}_{5}$.

Lemma 4.3. Suppose that $m<n<\omega$. Then

(i) $\mathbf{L}_{n} \vDash\{\langle n|| 0\rangle$,$\} and \mathbf{L}_{n} \not \models\langle m|$;

(ii) $\mathbf{R}_{n} \vDash\{\langle 0|| n\rangle$,$\} and \mathbf{R}_{n} \not \models|m\rangle$.

Consequently, the inclusions $\mathbf{L}_{m} \subset \mathbf{L}_{n}$ and $\mathbf{R}_{m} \subset \mathbf{R}_{n}$ hold and are strict.

\begin{tabular}{|c|c|c|c|c|c|c|c|c|c|c|c|c|}
\hline$P$ & 0 & $a$ & $b$ & $c$ & $d$ & $e$ & & & & & & \\
\hline 0 & 0 & 0 & 0 & 0 & 0 & 0 & $Q$ & 0 & $\frac{a}{0}$ & $\frac{b}{0}$ & $\begin{array}{l}c \\
\end{array}$ & $d$ \\
\hline$a$ & 0 & 0 & 0 & 0 & 0 & $b$ & 0 & 0 & 0 & o & 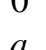 & \\
\hline$b$ & 0 & 0 & 0 & 0 & $b$ & $b$ & $\begin{array}{l}a \\
b\end{array}$ & 0 & $a$ & $h$ & 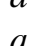 & $\begin{array}{l}a \\
0\end{array}$ \\
\hline$c$ & 0 & $a$ & $b$ & $c$ & 0 & 0 & $\begin{array}{l}b \\
C\end{array}$ & $\begin{array}{l}0 \\
0\end{array}$ & $\begin{array}{l}a \\
0\end{array}$ & $\begin{array}{l}0 \\
0\end{array}$ & $a_{c}$ & $c$ \\
\hline$d$ & 0 & 0 & 0 & 0 & $d$ & $d$ & $\begin{array}{l}c \\
d\end{array}$ & 0 & 0 & & $d$ & $d$ \\
\hline$e$ & 0 & 0 & 0 & 0 & & $e$ & & & & & & \\
\hline
\end{tabular}

PROOF. This is straightforward.

Let $P=\{0, a, b, c, d, e\}$ and $Q=\{0, a, b, c, d\}$ be the semigroups defined by the following multiplication tables:

The semigroup $P$ was brought to the author's attention by Volkov and the semigroup $Q$ appeared in [14] as $L C_{0}$. Let $\mathbf{P}$ and $\mathbf{Q}$ be the varieties generated by the semigroups $P$ and $Q$, respectively.

LEMMA 4.4.

(i) $\overline{\mathbf{A}}_{\mathbf{0}} \vDash\{\langle\widehat{\omega}|,| \widehat{\omega}\rangle\}$.

(ii) $P \in \overline{\mathbf{A}}_{\mathbf{0}} \cap \overline{\mathbf{B}}_{2}, P \vDash\{\langle\widehat{\omega}|| 0\rangle$,$\} , and P \not \models\langle\omega|$.

(iii) $Q \in \overline{\mathbf{B}}_{2}, Q \vDash|0\rangle$, and $Q \not \models\langle\widehat{\omega}|$. 
ProOF. (i) It easily follows from Lemma 2.1(i) that the variety $\overline{\mathbf{A}}_{\mathbf{0}}$ satisfies the identity $\alpha:\left(x^{2} y^{2} z^{2}\right)^{2} \approx x^{2} y^{2} z^{2}$. Then $\overline{\mathbf{A}}_{\mathbf{0}} \vDash\langle\widehat{\omega}|$ because

$$
\begin{aligned}
\left(h^{2} y^{2} x^{2}\right) y^{2} x^{2} & \stackrel{\alpha}{\approx}\left(h^{2} y^{2} x^{2} h^{2}\right) y^{2} x^{2} y^{2} x^{2} \\
& \stackrel{(3)}{\approx} h^{2} x^{2}\left(y^{2} h^{2} y^{2} x^{2} y^{2}\right) x^{2} \\
& \stackrel{(3)}{\approx}\left(h^{2} x^{2} y^{2} h^{2} x^{2} y^{2}\right) y^{2} x^{2} \\
& \stackrel{\alpha}{\approx} h^{2} x^{2}\left(y^{2} y^{2}\right) x^{2} \\
& (1) h^{2} x^{2} y^{2} x^{2} .
\end{aligned}
$$

The variety $\overline{\mathbf{A}}_{\mathbf{0}}$ is self-dual by Lemma 2.1(i) so that $\overline{\mathbf{A}}_{\mathbf{0}} \vDash|\widehat{\omega}\rangle$.

(ii) It is routine to show by Lemma 2.1(i) that $P \in \overline{\mathbf{A}}_{\mathbf{0}} \cap \overline{\mathbf{B}}_{\mathbf{2}}$ and $P \vDash\{\langle\widehat{\omega}|| 0\rangle$,$\} .$ Since $c^{2} a d^{2} e^{2} d^{2}=0 \neq b=c^{2} a e^{2} d^{2} e^{2} d^{2}$, it follows that $P \not \models\langle\omega|$.

(iii) It is routine to show by Lemma 2.1(i) that $Q \in \overline{\mathbf{B}}_{2}$ and $Q \vDash|0\rangle$. Since $b^{2} c^{2} d^{2} c^{2}=a \neq 0=b^{2} d^{2} c^{2} d^{2} c^{2}$, it follows that $Q \not \models\langle\widehat{\omega}|$.

Lemma 4.5. Let $i \in\{1,2,3,4\}$. Suppose that $\mathbf{V} \in \mathcal{I}_{i}$. Then $\mathbf{V}=\mathbf{E}_{i} \Sigma$ where $\mathbf{E}_{i}$ is the largest variety in $\mathcal{I}_{i}$ and $\Sigma \subseteq\{\langle\ell|| r\rangle$,$\} for some \ell, r \leq \widehat{\omega}$.

PROOF. There are four cases depending on the value of $i$.

CASE 1. $i=1$ so that $\mathbf{E}_{i}=\overline{\mathbf{A}}_{\mathbf{2}}$. It follows from [11, proof of Proposition 3.14] that if $\mathbf{V} \in \mathcal{I}_{1}$, then $\mathbf{V}=\overline{\mathbf{A}}_{\mathbf{2}} \Sigma$ for some set $\Sigma$ that contains some of the following identities:

$$
\mathbf{h}_{(\ell)} x^{2} y^{2} x^{2} \mathbf{t}_{(r)} \approx \mathbf{h}_{(\ell)} y^{2} x^{2} y^{2} x^{2} \mathbf{t}_{(r)}, \quad \mathbf{h}_{(\ell)} x^{2} y^{2} x^{2} \mathbf{t}_{(r)} \approx \mathbf{h}_{(\ell)} x^{2} y^{2} x^{2} y^{2} \mathbf{t}_{(r)} .
$$

It is easy to show that within the equational theory of $\mathbf{A}_{\mathbf{2}}$, the first identity in (6) is equivalent to $\langle\ell|$ independent of the value of $r$, and the second identity in (6) is equivalent to $|r\rangle$ independent of the value of $\ell$. It follows from Lemma 4.1 that the set $\Sigma$ can be chosen to be a subset of $\{\langle\ell|| r\rangle$,$\} for some \ell, r \leq \widehat{\omega}$.

CASE 2. $i=2$. The arguments in [11, proof of Proposition 3.14] can be repeated for varieties in $\mathcal{I}_{2}$. Therefore, the present case can be similarly established.

CASE 3. $i=3$ so that $\mathbf{E}_{i}=\overline{\mathbf{A}}_{\mathbf{0}}$. It follows from [11, proof of Proposition 4.3] that if $\mathbf{V} \in \mathcal{I}_{3}$, then $\mathbf{V}=\overline{\mathbf{A}}_{\mathbf{0}} \cap \mathbf{V}^{\prime}$ for some variety $\mathbf{V}^{\prime} \in \mathcal{I}_{1}$. It follows from Case 1 that $\mathbf{V}^{\prime}=\overline{\mathbf{A}}_{\mathbf{2}} \Sigma$ for some $\Sigma \subseteq\{\langle\ell|| r\rangle$,$\} with \ell, r \leq \widehat{\omega}$. Therefore, $\mathbf{V}=\overline{\mathbf{A}}_{\mathbf{0}} \cap \overline{\mathbf{A}}_{\mathbf{2}} \Sigma=\overline{\mathbf{A}}_{\mathbf{0}} \Sigma$ as required.

CASE 4. $i=4$ so that $\mathbf{E}_{i}=\overline{\mathbf{A}}_{\mathbf{0}} \cap \overline{\mathbf{B}}_{\mathbf{2}}$. Following [11, proof of Proposition 4.3], if $\mathbf{V} \in \mathcal{I}_{4}$, then $\mathbf{V}=\overline{\mathbf{A}}_{\mathbf{0}} \cap \overline{\mathbf{B}}_{\mathbf{2}} \cap \mathbf{V}^{\prime}$ for some variety $\mathbf{V}^{\prime} \in \mathcal{I}_{2}$. It follows from Case 2 that $\mathbf{V}^{\prime}=\overline{\mathbf{B}}_{\mathbf{2}} \Sigma$ for some $\Sigma \subseteq\{\langle\ell|| r\rangle$,$\} with \ell, r \leq \widehat{\omega}$. Therefore, $\mathbf{V}=\overline{\mathbf{A}}_{\mathbf{0}} \cap \overline{\mathbf{B}}_{\mathbf{2}} \cap \overline{\mathbf{B}}_{\mathbf{2}} \Sigma=$ $\left(\overline{\mathbf{A}}_{\mathbf{0}} \cap \overline{\mathbf{B}}_{2}\right) \Sigma$ as required. 
For the rest of this paper, it will be convenient to let

$$
\begin{gathered}
\left\langle\left.\ell\right|_{i}=\mathbf{E}_{i}\{\langle\ell|\},\right. \\
|r\rangle_{i}=\mathbf{E}_{i}\{|r\rangle\}, \\
\langle\ell, r\rangle_{i}=\mathbf{E}_{i}\{\langle\ell|,| r\rangle\}=\left\langle\left.\ell\right|_{i} \cap \mid r\right\rangle_{i},
\end{gathered}
$$

where $\ell, r \leq \widehat{\omega}$ and $\mathbf{E}_{i}$ is the largest variety in the interval $\mathcal{I}_{i}$ with $i \in\{1,2,3,4\}$.

LEMMA 4.6.

(i) $\langle 0,0\rangle_{1}=\mathbf{A}_{\mathbf{0}} \vee \mathbf{B}_{\mathbf{2}}$.

(ii) $\langle 0,0\rangle_{2}=\mathbf{A}_{\mathbf{0}}$.

(iii) $\langle 0,0\rangle_{3}=\mathbf{B}_{\mathbf{2}}$.

(iv) $\langle 0,0\rangle_{4}=\mathbf{B}_{\mathbf{0}}$.

Proof. (i) Since $\mathbf{A}_{\mathbf{0}} \vee \mathbf{B}_{\mathbf{2}}=\mathbf{A}_{\mathbf{2}}\left\{x^{2} y^{2} x^{2} \approx y^{2} x^{2} y^{2}\right\}$ by [14, Theorem 4.3(i)], it is routine to show that the varieties $\mathbf{A}_{\mathbf{0}} \vee \mathbf{B}_{\mathbf{2}}$ and $\langle 0,0\rangle_{1}$ coincide.

(ii) It follows from [14, Remark 4.1] that $\mathbf{A}_{\mathbf{0}}=\mathbf{A}_{\mathbf{2}}\{x y x y \approx y x y x\}$. It is routine to show that the semigroup $A_{0}$ satisfies the identities $x y^{2} x \approx x y x,\langle 0|$, and $|0\rangle$ so that the inclusion $\mathbf{A}_{\mathbf{0}} \subseteq\langle 0,0\rangle_{2}$ holds; the reverse inclusion holds since

$$
\begin{aligned}
x y x y & \stackrel{(2)}{\approx} x^{2} y^{2} x^{2} y^{2} \\
& \stackrel{\langle 0|}{\approx} y^{2} x^{2} y^{2} \\
& |0\rangle \\
& \approx y^{2} x^{2} y^{2} x^{2} \\
& (2) \\
& \approx y x y x .
\end{aligned}
$$

(iii) It follows from [14, proof of Proposition 3.5] that $\mathbf{B}_{\mathbf{2}}=\mathbf{A}_{\mathbf{2}}\left\{x^{2} y^{2} \approx y^{2} x^{2}\right\}$. It is then routine to show that this variety coincides with $\langle 0,0\rangle_{3}$.

(iv) This follows from parts (ii) and (iii) since $\mathbf{B}_{\mathbf{0}}=\mathbf{A}_{\mathbf{0}} \cap \mathbf{B}_{\mathbf{2}}$ by [8, Lemma 4.2 and Corollary 4.3].

\section{The intervals $\mathcal{I}_{1}, \mathcal{I}_{2}, \mathcal{I}_{3}$, and $\mathcal{I}_{4}$}

In every figure in this section, each $\bullet$ represents a finitely generated variety and each $\otimes$ represents a nonfinitely generated variety.

Proposition 5.1. The varieties in the interval $\mathcal{I}_{4}$ are shown in Figure 1.

PROOF. By Lemmas 4.4(i), 4.5, and 4.6(iv), the varieties in the interval $\mathcal{I}_{4}$ are possibly of the form $\left(\overline{\mathbf{A}}_{\mathbf{0}} \cap \overline{\mathbf{B}}_{2}\right) \Sigma$ where $\Sigma \subseteq\{\langle\ell|| r\rangle$,$\} for some \ell, r \leq \omega$. It follows from Lemma 4.1 that the locations of these varieties within the interval $\mathcal{I}_{4}$ are shown in Figure 1. It remains to verify that the varieties in Figure 1 are distinct and identify those that are finitely generated.

Consider the chain

$$
\mathbf{B}_{\mathbf{0}}=\langle 0,0\rangle_{4} \subseteq\langle 1,0\rangle_{4} \subseteq\langle 2,0\rangle_{4} \subseteq \cdots \subseteq\langle\omega, 0\rangle_{4} \subseteq|0\rangle_{4}
$$




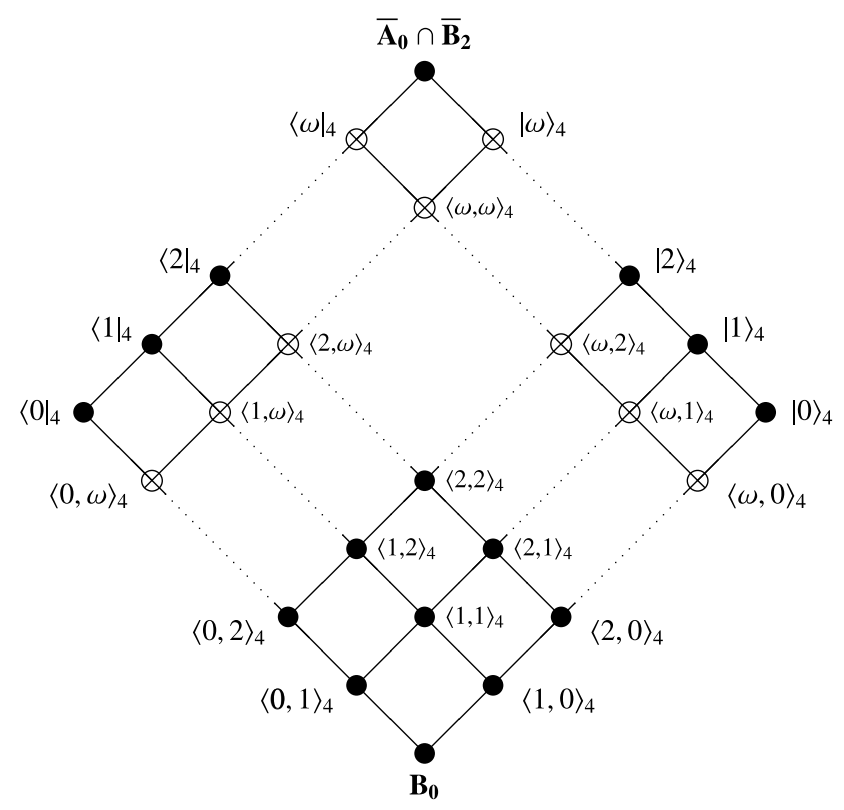

FIgURE 1. The interval $\mathcal{I}_{4}=\left[\mathbf{B}_{0}, \overline{\mathbf{A}}_{\mathbf{0}} \cap \overline{\mathbf{B}}_{2}\right]$.

in Figure 1. First suppose that $1 \leq \ell<\omega$. It follows from Lemma 4.3(i) that the variety $\mathbf{L}_{\ell}$ satisfies the identities $\langle\ell|$ and $|0\rangle$ but not the identity $\langle\ell-1|$, whence $\mathbf{B}_{\mathbf{0}} \vee \mathbf{L}_{\ell} \subseteq\langle\ell, 0\rangle_{4}$ and $\mathbf{B}_{\mathbf{0}} \vee \mathbf{L}_{\ell} \nsubseteq\langle\ell-1,0\rangle_{4}$. Therefore, the varieties $\mathbf{B}_{\mathbf{0}} \vee \mathbf{L}_{\ell}$ and $\langle\ell, 0\rangle_{4}$ must coincide. It follows that the varieties $\langle 0,0\rangle_{4},\langle 1,0\rangle_{4}, \ldots$ are distinct and finitely generated. Further, $\mathbf{L}_{\ell+1}$ is a subvariety of $\langle\omega, 0\rangle_{4}$ that is not contained in $\langle\ell, 0\rangle_{4}$. Hence, $\langle\omega, 0\rangle_{4} \nsubseteq\langle\ell, 0\rangle_{4}$ for all $\ell<\omega$. If the variety $\langle\omega, 0\rangle_{4}$ is generated by some finite semigroup $S$ of order $n$, then $S \vDash\langle n|$ by Lemma 4.2(i) so that the inclusion $\langle\omega, 0\rangle_{4} \subseteq\langle n, 0\rangle_{4}$ contradictorily holds. Therefore, the variety $\langle\omega, 0\rangle_{4}$ must be nonfinitely generated. Since the subsemigroup $\{0, b, c, d\}$ of $P$ is isomorphic to $B_{0}$, it follows from Lemma 4.4(ii) that $\mathbf{B}_{\mathbf{0}} \subseteq \mathbf{P} \subseteq|0\rangle_{4}$ and $\mathbf{P} \nsubseteq\langle\omega, 0\rangle_{4}$. Hence, $|0\rangle_{4}=\mathbf{P}$ is finitely generated.

Consequently, the varieties in (7) are distinct and, with the exception of $\langle\omega, 0\rangle_{4}$, are finitely generated. By symmetry, the varieties in the chain

$$
\mathbf{B}_{\mathbf{0}}=\langle 0,0\rangle_{4} \subseteq\langle 0,1\rangle_{4} \subseteq\langle 0,2\rangle_{4} \subseteq \cdots \subseteq\langle 0, \omega\rangle_{4} \subseteq\left\langle\left. 0\right|_{4}\right.
$$

are distinct and, with the exception of $\langle 0, \omega\rangle_{4}$, are finitely generated. It is routine to show that varieties of the form $\mathbf{U} \vee \mathbf{V}$, where $\mathbf{U}$ is from (7) and $\mathbf{V}$ is from (8), constitute the varieties in Figure 1. Distinctness and the finite generation property of the varieties in Figure 1 can then be verified easily. For instance, consider the variety $\langle\ell, r\rangle_{4}$. The inclusion

$$
\langle 0, r\rangle_{4} \vee\langle\ell, 0\rangle_{4} \subseteq\langle\ell, r\rangle_{4}
$$




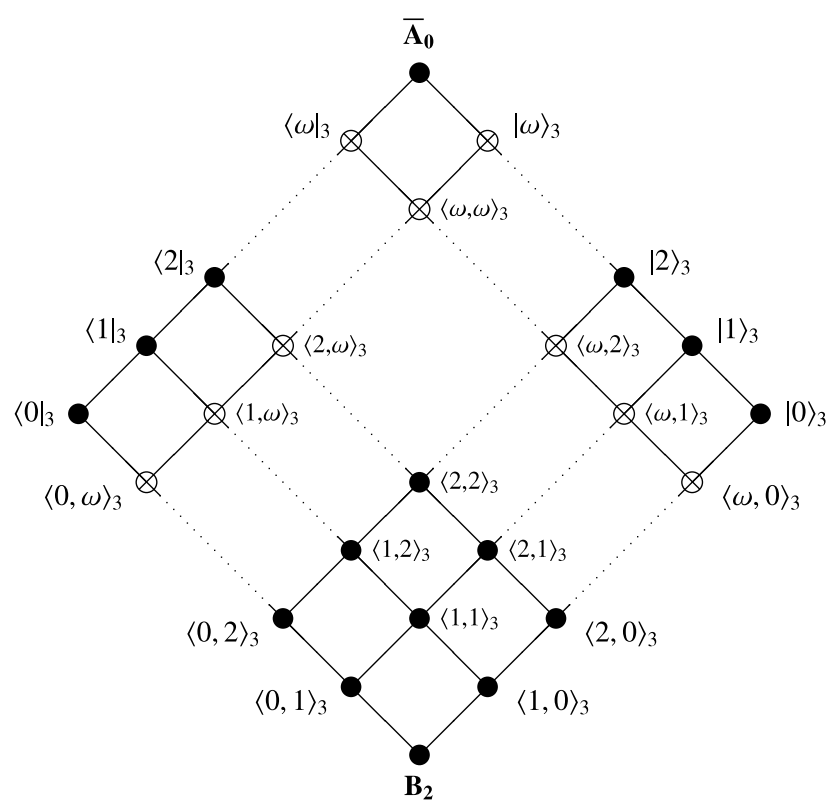

Figure 2. The interval $\mathcal{I}_{3}=\left[\mathbf{B}_{2}, \overline{\mathbf{A}}_{0}\right]$.

holds by Lemma 4.1. If $\ell, r<\omega$, then by Lemma 4.3, the subvarieties $\mathbf{L}_{\ell}$ and $\mathbf{R}_{r}$ of $\langle 0, r\rangle_{4} \vee\langle\ell, 0\rangle_{4}$ do not both belong to $\left\langle\ell^{\prime}, r^{\prime}\right\rangle_{4}$ whenever $\ell^{\prime}<\ell$ or $r^{\prime}<r$, whence equality must hold in (9). If either $\ell=\omega$ or $r=\omega$, then Lemma 4.2 can be used to show that $\langle\ell, r\rangle_{4}$ is nonfinitely generated and hence distinct from all its subvarieties in Figure 1. Similar arguments can be applied to the remaining varieties $\left\langle\left.\ell\right|_{4}, \mid r\right\rangle_{4}$, and $\overline{\mathbf{A}}_{\mathbf{0}} \cap \overline{\mathbf{B}}_{\mathbf{2}}$.

PROPOSITION 5.2. The varieties in the interval $\mathcal{I}_{3}$ are shown in Figure 2.

PROOF. This is almost identical to the proof of Proposition 5.1; the biggest difference lies in showing that the variety $|0\rangle_{3}$ in the chain

$$
\mathbf{B}_{2}=\langle 0,0\rangle_{3} \subseteq\langle 1,0\rangle_{3} \subseteq\langle 2,0\rangle_{3} \subseteq \cdots \subseteq\langle\omega, 0\rangle_{3} \subseteq|0\rangle_{3}
$$

is finitely generated. Recall that in the proof of Proposition 5.1, it was shown that $|0\rangle_{4}=\mathbf{P}$. In the present case, the variety $\mathbf{B}_{\mathbf{2}} \vee \mathbf{P}$ should be used instead. It follows from Lemma 4.4(ii) that $\mathbf{B}_{\mathbf{2}} \vee \mathbf{P} \subseteq|0\rangle_{3}$ and $\mathbf{B}_{\mathbf{2}} \vee \mathbf{P} \nsubseteq\langle\omega, 0\rangle_{3}$. Therefore, $|0\rangle_{3}=\mathbf{B}_{\mathbf{2}} \vee \mathbf{P}$ is finitely generated.

PROPOSITION 5.3. The varieties in the interval $\mathcal{I}_{2}$ are shown in Figure 3.

PROOF. By Lemmas 4.5 and 4.6(ii), the varieties in the interval $\mathcal{I}_{2}$ are possibly of the form $\overline{\mathbf{B}}_{2} \Sigma$ where $\Sigma \subseteq\{\langle\ell|| r\rangle$,$\} for some \ell, r \leq \widehat{\omega}$. It follows from Lemma 4.1 that 


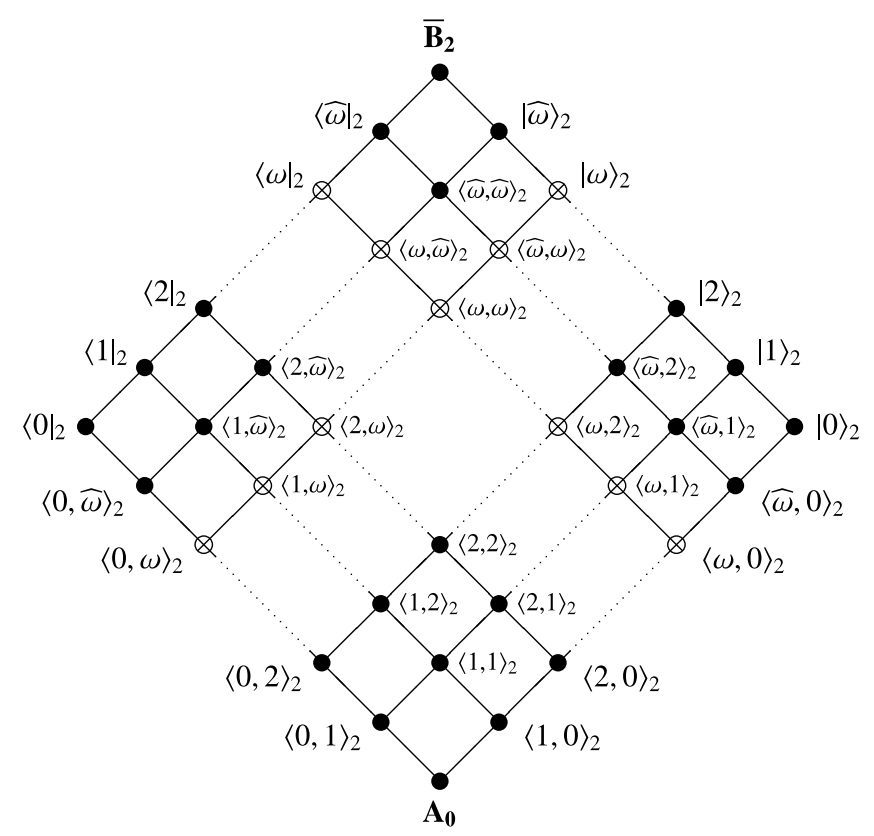

FIgURE 3. The interval $\mathcal{I}_{2}=\left[\mathbf{A}_{0}, \overline{\mathbf{B}}_{2}\right]$.

the locations of these varieties within the lattice $\mathcal{I}_{2}$ are shown in Figure 3 . It remains to verify that the varieties in Figure 3 are distinct and identify those that are finitely generated.

Consider the chain

$$
\mathbf{A}_{\mathbf{0}}=\langle 0,0\rangle_{2} \subseteq\langle 1,0\rangle_{2} \subseteq\langle 2,0\rangle_{2} \subseteq \cdots \subseteq\langle\omega, 0\rangle_{2} \subseteq\langle\widehat{\omega}, 0\rangle_{2} \subseteq|0\rangle_{2}
$$

in Figure 3. Following the arguments in the proof of Proposition 5.1, the varieties $\langle 0,0\rangle_{2},\langle 1,0\rangle_{2}, \ldots$ are distinct and finitely generated, and the variety $\langle\omega, 0\rangle_{2}$ is nonfinitely generated. It follows from Lemma 4.4(ii) that $\mathbf{A}_{\mathbf{0}} \subseteq \mathbf{A}_{\mathbf{0}} \vee \mathbf{P} \subseteq\langle\widehat{\omega}, 0\rangle_{2}$ and $\mathbf{A}_{\mathbf{0}} \vee \mathbf{P} \nsubseteq\langle\omega, 0\rangle_{2}$. Hence, $\langle\widehat{\omega}, 0\rangle_{2}=\mathbf{A}_{\mathbf{0}} \vee \mathbf{P}$ is finitely generated. Since the subsemigroup $\{0, a, b, c\}$ of $Q$ is isomorphic to $A_{0}$, it follows from Lemma 4.4(iii) that $\mathbf{A}_{\mathbf{0}} \subseteq \mathbf{Q} \subseteq|0\rangle_{2}$ and $\mathbf{Q} \nsubseteq\langle\widehat{\omega}, 0\rangle_{2}$. Therefore, $|0\rangle_{2}=\mathbf{Q}$ is finitely generated. Consequently, the varieties in (10) are distinct and, with the exception of $\langle\omega, 0\rangle_{2}$, are finitely generated. By symmetry, the varieties in the chain

$$
\mathbf{A}_{\mathbf{0}}=\langle 0,0\rangle_{2} \subseteq\langle 0,1\rangle_{2} \subseteq\langle 0,2\rangle_{2} \subseteq \cdots \subseteq\langle 0, \omega\rangle_{2} \subseteq\langle 0, \widehat{\omega}\rangle_{2} \subseteq\left\langle\left. 0\right|_{2}\right.
$$

are distinct and, with the exception of $\langle 0, \omega\rangle_{2}$, are finitely generated. The rest of the present proof follows in the same manner as the proof of Proposition 5.1.

PROPOSITION 5.4. The varieties in the interval $\mathcal{I}_{1}$ are shown in Figure 4. 


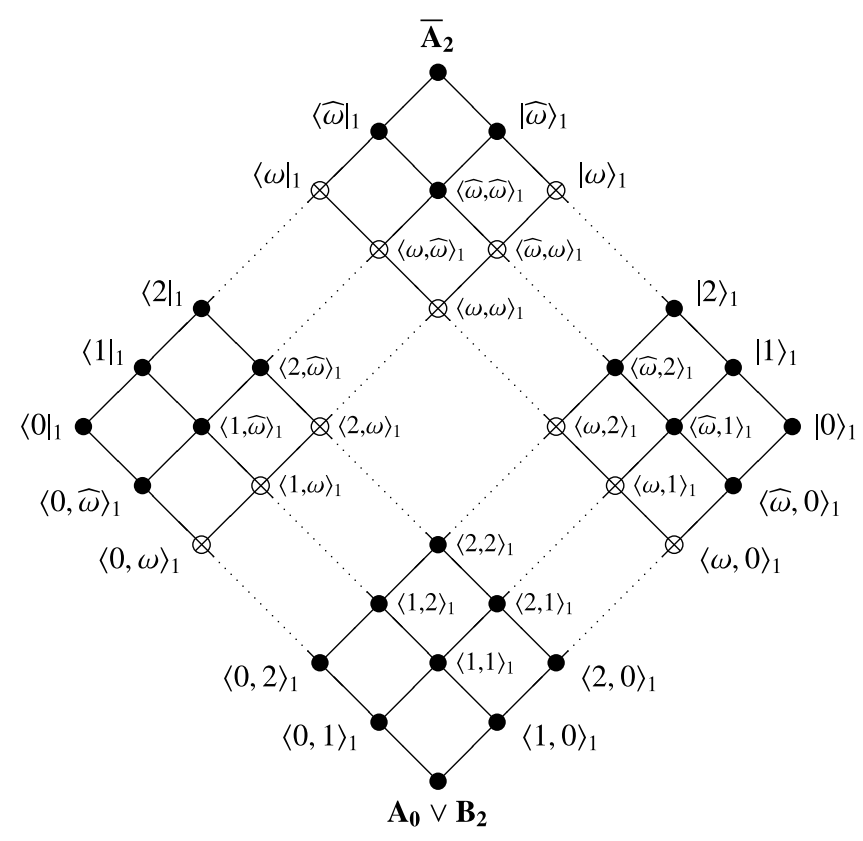

FIGURE 4. The interval $\mathcal{I}_{1}=\left[\mathbf{A}_{0} \vee \mathbf{B}_{2}, \overline{\mathbf{A}}_{2}\right]$.

PROOF. This is similar to Proposition 5.3 in the manner Proposition 5.2 is similar to Proposition 5.1.

REMARK 3. The varieties $\left\langle\left. 0\right|_{1}, \mid 0\right\rangle_{1},\left\langle\left. 0\right|_{2}, \mid 0\right\rangle_{2}, \overline{\mathbf{A}}_{\mathbf{2}}$, and $\overline{\mathbf{B}}_{\mathbf{2}}$ have previously been shown to be finitely generated in [14, Theorems 4.2 and 4.3].

LEMMA 5.5.

(i) The variety $\overline{\mathbf{A}}_{\mathbf{2}}$ is the only subvariety of $\mathbf{A}_{\mathbf{2}}$ that covers the variety $\overline{\mathbf{B}}_{\mathbf{2}}$.

(ii) The variety $\langle\widehat{\omega}, \widehat{\omega}\rangle_{1}$ is the only subvariety of $\mathbf{A}_{2}$ that covers the variety $\overline{\mathbf{A}}_{\mathbf{0}}$.

(iii) The varieties $\langle\widehat{\omega}, \widehat{\omega}\rangle_{2}$ and $\overrightarrow{\mathbf{A}}_{\mathbf{0}}$ are the only subvarieties of $\mathbf{A}_{2}$ that cover the variety $\overline{\mathbf{A}}_{\mathbf{0}} \cap \overline{\mathbf{B}}_{\mathbf{2}}$.

Proof. (i) The variety $\overline{\mathbf{B}}_{\mathbf{2}}$ contains the semigroup $A_{0}$ and so is not a subvariety of $\overline{\mathbf{A}}_{\mathbf{0}}$. It follows from Lemmas 2.1(ii) and 2.3 that within the lattice $\mathcal{L}\left(\mathbf{A}_{\mathbf{2}}\right)$, the variety $\overline{\mathbf{B}}_{\mathbf{2}}$ can only be covered by some variety from the interval $\mathcal{I}_{1}$. The inclusion $\overline{\mathbf{B}}_{\mathbf{2}} \subseteq \overline{\mathbf{A}}_{\mathbf{2}}$ follows from Lemma 2.1(ii). Since the variety $\overline{\mathbf{B}}_{\mathbf{2}}$ is self-dual by Lemma 2.1(i), it does not satisfy the identities $\langle\widehat{\omega}|$ and $|\widehat{\omega}\rangle$ by Lemma 4.4(iii). It follows from Proposition 5.4 that $\overline{\mathbf{B}}_{2}$ is not contained in any proper subvariety of $\overline{\mathbf{A}}_{2}$ in $\mathcal{I}_{1}$, whence $\overline{\mathbf{A}}_{2}$ is the only subvariety of $\mathbf{A}_{\mathbf{2}}$ that covers $\overline{\mathbf{B}}_{\mathbf{2}}$.

(ii) The variety $\overline{\mathbf{A}}_{\mathbf{0}}$ contains the semigroup $B_{2}$ and so is not a subvariety of $\overline{\mathbf{B}}_{\mathbf{2}}$. It follows from Lemmas 2.1 (ii) and 2.3 that within the lattice $\mathcal{L}\left(\mathbf{A}_{\mathbf{2}}\right)$, the variety $\overline{\mathbf{A}}_{\mathbf{0}}$ can only be covered by some variety from $\mathcal{I}_{1}$. The inclusion $\overline{\mathbf{A}}_{\mathbf{0}} \subseteq\langle\widehat{\omega}, \widehat{\omega}\rangle_{1}$ follows from 
Lemmas 2.1(ii) and 4.4(i). Since the variety $\overline{\mathbf{A}}_{\mathbf{0}}$ is self-dual by Lemma 2.1(i), it does not satisfy the identities $\langle\omega|$ and $|\omega\rangle$ by Lemma 4.4(ii). It follows from Proposition 5.4 that $\overline{\mathbf{A}}_{\mathbf{0}}$ is not contained in any proper subvariety of $\langle\widehat{\omega}, \widehat{\omega}\rangle_{1}$ in $\mathcal{I}_{1}$, whence $\langle\widehat{\omega}, \widehat{\omega}\rangle_{1}$ is the only subvariety of $\mathbf{A}_{2}$ that covers $\overline{\mathbf{A}}_{\mathbf{0}}$.

(iii) The variety $\overline{\mathbf{A}}_{\mathbf{0}} \cap \overline{\mathbf{B}}_{\mathbf{2}}$ contains the semigroup $B_{0}$ and so is not a subvariety of $\overline{\mathbf{B}}_{\mathbf{0}}$. It follows from Lemmas 2.1 (ii) and 2.3 that within the lattice $\mathcal{L}\left(\mathbf{A}_{\mathbf{2}}\right)$, the variety $\overline{\mathbf{A}}_{\mathbf{0}} \cap \overline{\mathbf{B}}_{\mathbf{2}}$ can only be covered by some variety from $\mathcal{I}_{1} \cup \mathcal{I}_{2} \cup \mathcal{I}_{3}$. If $\overline{\mathbf{A}}_{\mathbf{0}} \cap \overline{\mathbf{B}}_{\mathbf{2}}$ is a subvariety of some variety $\mathbf{V}$ in $\mathcal{I}_{1}$, then $\overline{\mathbf{A}}_{\mathbf{0}} \cap \overline{\mathbf{B}}_{2}$ is a subvariety of $\mathbf{V} \cap \overline{\mathbf{B}}_{2}$ in $\mathcal{I}_{2}$. Therefore, $\overline{\mathbf{A}}_{\mathbf{0}} \cap \overline{\mathbf{B}}_{\mathbf{2}}$ can only be covered by some variety from $\mathcal{I}_{2} \cup \mathcal{I}_{3}$. The inclusion $\overline{\mathbf{A}}_{\mathbf{0}} \cap \overline{\mathbf{B}}_{\mathbf{2}} \subseteq\langle\widehat{\omega}, \widehat{\omega}\rangle_{2}$ follows from Lemmas 2.1(ii) and 4.4(i), and the inclusion $\overline{\mathbf{A}}_{\mathbf{0}} \cap \overline{\mathbf{B}}_{\mathbf{2}} \subseteq \overline{\mathbf{A}}_{\mathbf{0}}$ holds vacuously. Since the variety $\overline{\mathbf{A}}_{\mathbf{0}} \cap \overline{\mathbf{B}}_{\mathbf{2}}$ is self-dual by Lemma 2.1(i), it does not satisfy the identities $\langle\omega|$ and $|\omega\rangle$ by Lemma 4.4(ii). It follows from Propositions 5.2 and 5.3 that the variety $\overline{\mathbf{A}}_{\mathbf{0}} \cap \overline{\mathbf{B}}_{\mathbf{2}}$ is neither contained in any proper subvariety of $\langle\widehat{\omega}, \widehat{\omega}\rangle_{2}$ in $\mathcal{I}_{2}$ nor contained in any proper subvariety of $\overline{\mathbf{A}}_{\mathbf{0}}$ in $\mathcal{I}_{3}$, whence $\langle\widehat{\omega}, \widehat{\omega}\rangle_{2}$ and $\overline{\mathbf{A}}_{\mathbf{0}}$ are the only subvarieties of $\mathbf{A}_{\mathbf{2}}$ that cover $\overline{\mathbf{A}}_{\mathbf{0}} \cap \overline{\mathbf{B}}_{\mathbf{2}}$.

By Lemma 5.5 and methods similar to its proof, it is routine to show how the four intervals $\mathcal{I}_{1}, \ldots, \mathcal{I}_{4}$, together with the variety $\mathbf{A}_{2}$, combine to form the single interval $\left[\mathbf{B}_{\mathbf{0}}, \mathbf{A}_{\mathbf{2}}\right]$ in Figure 5. To avoid overcrowding the figure, most of the varieties are unlabelled and most of the following coverings are not shown:

(a) $\quad \overline{\mathbf{B}}_{\mathbf{2}} \prec \overline{\mathbf{A}}_{\mathbf{2}}$ and $\overline{\mathbf{A}}_{\mathbf{0}} \cap \overline{\mathbf{B}}_{\mathbf{2}} \prec \overline{\mathbf{A}}_{\mathbf{0}}$;

(b) $\left\langle\left.\ell\right|_{2} \prec\left\langle\left.\ell\right|_{1}, \mid r\right\rangle_{2} \prec \mid r\right\rangle_{1}$, and $\langle\ell, r\rangle_{2} \prec\langle\ell, r\rangle_{1}$ for all $\ell, r \leq \widehat{\omega}$;

(c) $\left\langle\left.\ell\right|_{4} \prec\left\langle\left.\ell\right|_{3}, \mid r\right\rangle_{4} \prec \mid r\right\rangle_{3}$, and $\langle\ell, r\rangle_{4} \prec\langle\ell, r\rangle_{3}$ for all $\ell, r \leq \omega$.

Any unlabelled variety in Figure 5 can easily be identified by referring to Figures 1-4. It is easy to see that the interval $\left[\mathbf{B}_{\mathbf{0}}, \mathbf{A}_{\mathbf{2}}\right]$ is a distributive lattice.

COROllary 5.6.

(i) $\quad \mathbf{B}_{\mathbf{0}} \subset\langle\omega, 0\rangle_{4} \subset|0\rangle_{4}$.

(ii) $\langle\omega, 0\rangle_{4}=\mathbf{B}_{\mathbf{0}} \vee \bigvee\left\{\mathbf{L}_{n} \mid n<\omega\right\}$.

(iii) $|0\rangle_{4}=\mathbf{P}$.

PROOF. The inclusions in part (i) have been established in Figure 1. It has been shown in the proof of Proposition 5.1 that $|0\rangle_{4}=\mathbf{P}$ and $\langle n, 0\rangle_{4}=\mathbf{B}_{\mathbf{0}} \vee \mathbf{L}_{n}$ for any $n<\omega$. Hence, $\langle\omega, 0\rangle_{4}=\bigvee\left\{\langle n, 0\rangle_{4} \mid n<\omega\right\}=\mathbf{B}_{\mathbf{0}} \vee \bigvee\left\{\mathbf{L}_{n} \mid n<\omega\right\}$.

\section{The word problems of $B_{0},\langle\omega, 0\rangle_{4}$, and $|0\rangle_{4}$}

Any words $\mathbf{w}_{1}, \ldots, \mathbf{w}_{n}$ are said to be disjoint if the sets $\mathrm{C}\left(\mathbf{w}_{1}\right), \ldots, \mathbf{C}\left(\mathbf{w}_{n}\right)$ are pairwise disjoint. A word of length at least 2 is connected if it cannot be written as a product of two disjoint nonempty words. Any word $\mathbf{u}$ can be uniquely written in natural form, that is,

$$
\mathbf{u}=\prod_{i=1}^{m}\left(\mathbf{s}_{i} \mathbf{u}_{i}\right)
$$




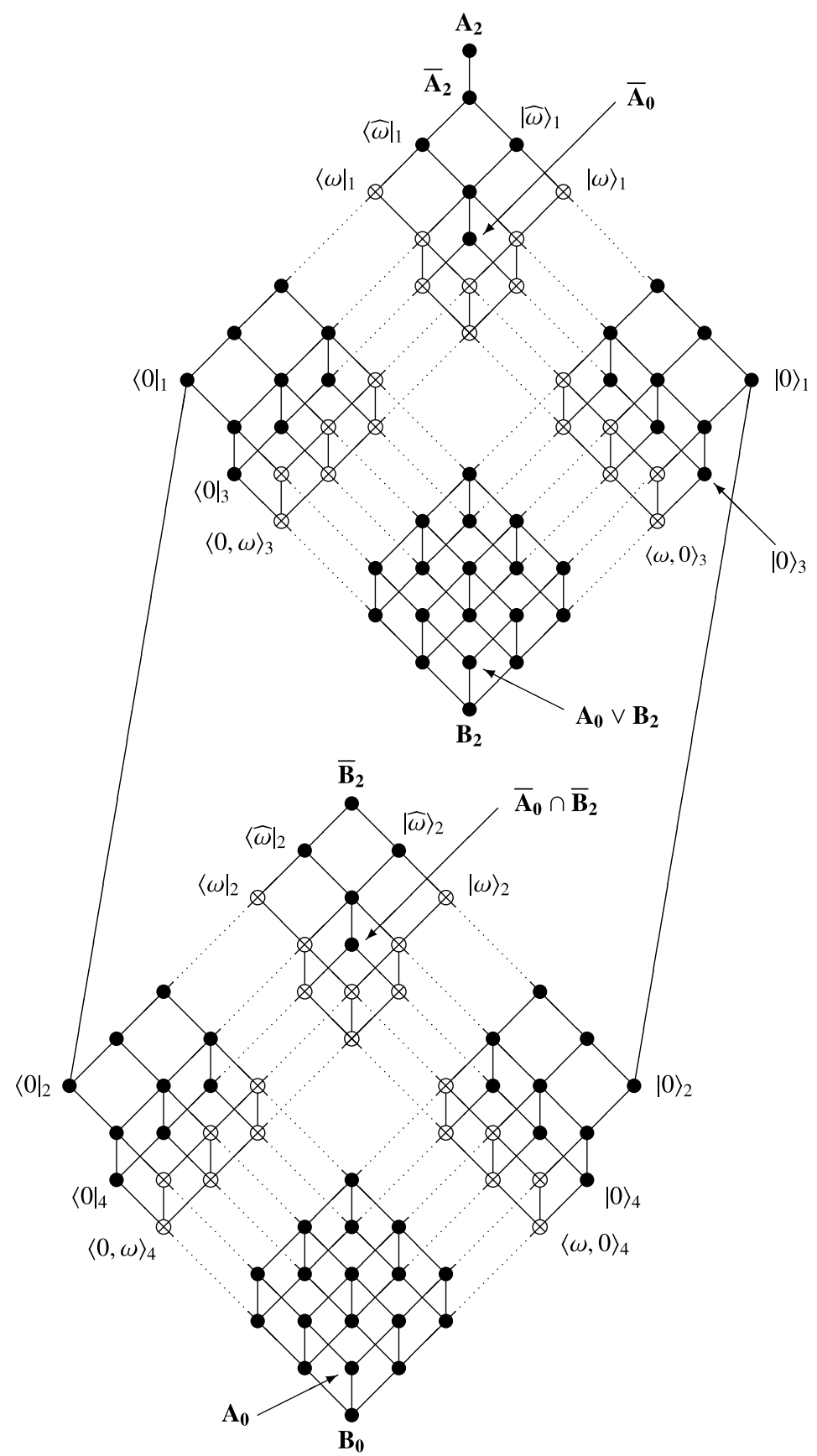

FIGURE 5. The interval $\left[\mathbf{B}_{\mathbf{0}}, \mathbf{A}_{\mathbf{2}}\right]$. 
where each $\mathbf{s}_{i}$ is a simple word with $\mathbf{s}_{1}$ possibly being empty, each $\mathbf{u}_{i}$ is a product of connected words with $\mathbf{u}_{m}$ possibly being empty, and the words $\mathbf{s}_{1}, \mathbf{u}_{1}, \ldots, \mathbf{s}_{m}, \mathbf{u}_{m}$ are disjoint.

LEMMA 6.1. Let

$$
\mathbf{u}=\prod_{i=1}^{m}\left(\mathbf{s}_{i} \mathbf{u}_{i}\right) \quad \text { and } \quad \mathbf{v}=\prod_{i=1}^{n}\left(\mathbf{t}_{i} \mathbf{v}_{i}\right)
$$

be words written in natural form. Then $\mathbf{B}_{\mathbf{0}} \vDash \mathbf{u} \approx \mathbf{v}$ if and only if $m=n, \mathbf{s}_{i}=\mathbf{t}_{i}$, and $\mathrm{C}\left(\mathbf{u}_{i}\right)=\mathrm{C}\left(\mathbf{v}_{i}\right)$ for all $i$.

PROOF. This follows from the proof of part 3 of the first proposition in [4].

The head and tail of a word $\mathbf{w}$, denoted by $\mathrm{h}(\mathbf{w})$ and $\mathrm{t}(\mathbf{w})$, are the first and last letters occurring in $\mathbf{w}$, respectively.

LEMMA 6.2. Let $\mathbf{u} \approx \mathbf{v}$ be any identity of the variety $\mathbf{B}_{\mathbf{0}}$, say

$$
\mathbf{u}=\prod_{i=1}^{m}\left(\mathbf{s}_{i} \mathbf{u}_{i}\right) \quad \text { and } \quad \mathbf{v}=\prod_{i=1}^{m}\left(\mathbf{s}_{i} \mathbf{v}_{i}\right)
$$

with $\mathrm{C}\left(\mathbf{u}_{i}\right)=\mathrm{C}\left(\mathbf{v}_{i}\right)$ for all $i$ when $\mathbf{u}$ and $\mathbf{v}$ are written in natural form. Then

(i) $|0\rangle_{4} \vDash \mathbf{u} \approx \mathbf{v}$ if and only if $\mathrm{h}\left(\mathbf{u}_{i}\right)=\mathrm{h}\left(\mathbf{v}_{i}\right)$ for all $i$;

(ii) $\langle\omega, 0\rangle_{4} \vDash \mathbf{u} \approx \mathbf{v}$ if and only if $\mathrm{h}\left(\mathbf{u}_{1}\right)=\mathrm{h}\left(\mathbf{v}_{1}\right)$.

ProOf. (i) Suppose that $\mathrm{h}\left(\mathbf{u}_{j}\right) \neq \mathrm{h}\left(\mathbf{v}_{j}\right)$ for some $j$. Let $\varphi$ be the following substitution into the semigroup $P$ of $|0\rangle_{4}$ :

$$
x \mapsto \begin{cases}c & \text { if } x \text { occurs before } \mathrm{t}\left(\mathbf{s}_{j}\right) \text { in } \mathbf{u}, \\ a & \text { if } x=\mathrm{t}\left(\mathbf{s}_{j}\right), \\ d & \text { if } x=\mathrm{h}\left(\mathbf{u}_{j}\right) \\ e & \text { if } x \text { occurs after } \mathrm{h}\left(\mathbf{u}_{j}\right) \text { in } \mathbf{u} .\end{cases}
$$

Then

$$
\mathbf{u} \varphi=\left\{\begin{array}{ll}
d & \text { if } j=1 \text { and } \mathbf{s}_{1}=\emptyset, \\
0 & \text { if } \mathbf{s}_{1} \neq \varnothing,
\end{array} \quad \text { and } \quad \mathbf{v} \varphi= \begin{cases}e & \text { if } j=1 \text { and } \mathbf{s}_{1}=\emptyset \\
b & \text { if } \mathbf{s}_{1} \neq \varnothing\end{cases}\right.
$$

Therefore, $P \not \models \mathbf{u} \approx \mathbf{v}$, whence $|0\rangle_{4} \not \models \mathbf{u} \approx \mathbf{v}$ by Corollary 5.6(iii).

Conversely, suppose that $\mathrm{h}\left(\mathbf{u}_{i}\right)=\mathrm{h}\left(\mathbf{v}_{i}\right)$ for all $i$. The variety $|0\rangle_{4}$ is a subvariety of $\overline{\mathbf{B}}_{\mathbf{2}}$ and so satisfies the identities (2). It is routine to show that the semigroup $P$ satisfies the identities

$$
x^{3} \approx x^{2}, \quad x^{2} y^{2} z^{2} \approx x^{2} z^{2} y^{2}
$$

so that by Corollary 5.6(iii), the variety $|0\rangle_{4}$ also satisfies these identities. Therefore, in order to show that $|0\rangle_{4} \vDash \mathbf{u} \approx \mathbf{v}$, it suffices to show that every $\mathbf{u}_{i} \approx \mathbf{v}_{i}$ is a consequence 
of the identities $\{(2),(11)\}$. Let $\mathrm{C}\left(\mathbf{u}_{i}\right)=\mathrm{C}\left(\mathbf{v}_{i}\right)=\left\{x_{1}, \ldots, x_{k}\right\}$ with $\mathrm{h}\left(\mathbf{u}_{i}\right)=\mathrm{h}\left(\mathbf{v}_{i}\right)=$ $x_{1}$. Let $\chi$ be the substitution $x \mapsto x^{2}$ for all $x \in \mathcal{X}$. Since $\mathbf{u}_{i}$ is product of connected words, any simple letter in $\mathbf{u}_{i}$ must occur in some factor of $\mathbf{u}_{i}$ that begins and ends with a common letter. It follows that the identity $\mathbf{u}_{i} \approx \mathbf{u}_{i} \chi$ is a consequence of the identities (2). Since $\mathbf{u}_{i} \chi$ is a word over $\left\{x_{1}^{2}, \ldots, x_{k}^{2}\right\}$ that begins with $x_{1}^{2}$, it is easy to show that the identity $\mathbf{u}_{i} \chi \approx x_{1}^{2} \cdots x_{k}^{2}$ is a consequence of the identities (11). Hence, the identity $\mathbf{u}_{i} \approx x_{1}^{2} \cdots x_{k}^{2}$ is a consequence of the identities $\{(2),(11)\}$. By the same argument, the identity $\mathbf{v}_{i} \approx x_{1}^{2} \cdots x_{k}^{2}$ is also a consequence of the identities $\{(2),(11)\}$. Therefore, the identity $\mathbf{u}_{i} \approx \mathbf{v}_{i}$ is a consequence of the identities $\{(2),(11)\}$ as required.

(ii) Suppose that $\mathrm{h}\left(\mathbf{u}_{1}\right) \neq \mathrm{h}\left(\mathbf{v}_{1}\right)$. Let $\ell=\left|\mathbf{s}_{1}\right|+1$. It is easy to show that the identity $\mathbf{u} \approx \mathbf{v}$ is not a consequence of the identities $\left\{(1), \lambda_{\ell}\right\}$ that define the variety $\mathbf{L}_{\ell}$. It follows from Corollary 5.6(ii) that $\langle\omega, 0\rangle_{4} \not \models \mathbf{u} \approx \mathbf{v}$.

Conversely, suppose that $\mathrm{h}\left(\mathbf{u}_{1}\right)=\mathrm{h}\left(\mathbf{v}_{1}\right)=h$. Let $n \in\{1,2, \ldots\}$. Since $\mathbf{u}_{1}$ is a product of connected words, the letter $h$ is nonsimple in $\mathbf{u}_{1}$ so that the identity $\mathbf{u}_{1} \approx h^{n} \mathbf{u}_{1}$ is a consequence of the identities (2). Similarly, the identity $\mathbf{v}_{1} \approx h^{n} \mathbf{v}_{1}$ is also a consequence of the identities (2). Since

$$
\begin{aligned}
& \mathbf{u}=\mathbf{s}_{1} \mathbf{u}_{1} \prod_{i=2}^{m}\left(\mathbf{s}_{i} \mathbf{u}_{i}\right) \\
& \stackrel{(2)}{\approx} \mathbf{s}_{1}\left(h^{n} \mathbf{u}_{1} \prod_{i=2}^{m}\left(\mathbf{s}_{i} \mathbf{u}_{i}\right)\right) \\
& \stackrel{\lambda_{n}}{\approx} \mathbf{s}_{1}\left(h^{n} \mathbf{v}_{1}\right) \prod_{i=2}^{m}\left(\mathbf{s}_{i} \mathbf{v}_{i}\right) \\
& \stackrel{(2)}{\approx} \mathbf{s}_{1} \mathbf{v}_{1} \prod_{i=2}^{m}\left(\mathbf{s}_{i} \mathbf{v}_{i}\right) \\
&=\mathbf{v}
\end{aligned}
$$

the identity $\mathbf{u} \approx \mathbf{v}$ is a consequence of the identities $\left\{(2), \lambda_{n}\right\}$. The variety $\mathbf{L}_{n}$, being a subvariety of $\overline{\mathbf{B}}_{\mathbf{0}}$, must satisfy the identities (2) and so also the identity $\mathbf{u} \approx \mathbf{v}$. Since $n \in\{1,2, \ldots\}$ is arbitrary, every variety $\mathbf{L}_{n}$ satisfies the identity $\mathbf{u} \approx \mathbf{v}$. It follows from Corollary 5.6(ii) that $\langle\omega, 0\rangle_{4} \vDash \mathbf{u} \approx \mathbf{v}$.

LEMMA 6.3. Writing a word $\mathbf{w}$ in natural form is a problem with complexity $\mathcal{O}(|\mathbf{w}|)$.

PROOF. Any word $\mathbf{w}$ can always be uniquely decomposed in the form $\mathbf{w}=\mathbf{w}_{1} \cdots \mathbf{w}_{m}$ where $\mathbf{w}_{1}, \ldots, \mathbf{w}_{m}$ are disjoint words, each of which is either connected or of length 1 . This decomposition of $\mathbf{w}$ is referred to as the canonical decomposition of $\mathbf{w}$. It has been shown in [14, Section 2] that any word $\mathbf{w}$ corresponds to some directed graph $G(\mathbf{w})$ where the canonical decomposition $\mathbf{w}=\mathbf{w}_{1} \cdots \mathbf{w}_{m}$ corresponds to the ordered list $\left(G\left(\mathbf{w}_{1}\right), \ldots, G\left(\mathbf{w}_{m}\right)\right)$ of strongly connected components of $G(\mathbf{w})$.

Tarjan [24] demonstrated that decomposing a directed graph $G=(V, E)$ into its strongly connected components is a problem with complexity $\mathcal{O}(|V|+|E|)$. 
For the directed graph $G(\mathbf{w})$, the numbers of vertices and edges are bounded by $|\mathbf{w}|$. Therefore, finding the canonical decomposition of a word $\mathbf{w}$ is a problem with complexity $\mathcal{O}(|\mathbf{w}|)$. Once a canonical decomposition $\mathbf{w}=\mathbf{w}_{1} \cdots \mathbf{w}_{m}$ is obtained, writing $\mathbf{w}$ in natural form amounts to gathering consecutive factors from $\mathbf{w}_{1}, \ldots, \mathbf{w}_{m}$ of length 1 into simple words and gathering consecutive factors that are connected. Consequently, writing a word $\mathbf{w}$ in natural form is a problem with complexity $\mathcal{O}(|\mathbf{w}|)$.

Proposition 6.4. Let $\mathbf{V} \in\left\{\mathbf{B}_{\mathbf{0}},\langle\omega, 0\rangle_{4},|0\rangle_{4}\right\}$ and let $\mathbf{u} \approx \mathbf{v}$ be any identity where $\mathbf{u}$ and $\mathbf{v}$ are words of length at most $k$. Then deciding if the condition $\mathbf{V} \vDash \mathbf{u} \approx \mathbf{v}$ holds is a problem with complexity $\mathcal{O}(k)$.

PROOF. By Lemma 6.3, writing the words $\mathbf{u}$ and $\mathbf{v}$ in natural form is a problem with complexity $\mathcal{O}(k)$. Then it is clear by Lemma 6.1 that deciding if the condition $\mathbf{B}_{\mathbf{0}} \vDash$ $\mathbf{u} \approx \mathbf{v}$ holds has complexity $\mathcal{O}(k)$. If $\mathbf{B}_{\mathbf{0}} \not \models \mathbf{u} \approx \mathbf{v}$, then both $\langle\omega, 0\rangle_{4} \not \models \mathbf{u} \approx \mathbf{v}$ and $|0\rangle_{4} \not \models$ $\mathbf{u} \approx \mathbf{v}$ by Corollary 5.6(i). If $\mathbf{B}_{\mathbf{0}} \vDash \mathbf{u} \approx \mathbf{v}$, then by Lemma 6.2, deciding if the conditions $\langle\omega, 0\rangle_{4} \vDash \mathbf{u} \approx \mathbf{v}$ and $|0\rangle_{4} \vDash \mathbf{u} \approx \mathbf{v}$ hold are problems with complexity $\mathcal{O}(k)$.

\section{Proofs of the main results}

Let $\mathcal{F}$ and $\mathcal{S}$ be the sets of subvarieties of $\mathbf{A}_{2}$ that are finitely generated and small, respectively.

LEMMA 7.1. $\mathcal{S}=\mathcal{F} \cap \mathcal{I}_{5}$.

Proof. All varieties in the interval $\left[\mathbf{B}_{\mathbf{0}}, \mathbf{A}_{\mathbf{2}}\right]$ are nonsmall since the variety $\mathbf{B}_{\mathbf{0}}$ is nonsmall [9]. Therefore, the inclusion $\mathcal{S} \subseteq \mathcal{I}_{5}$ holds and the present lemma follows from Proposition 3.3.

Proof of Theorem A. (i) The inclusion $\mathcal{S} \subseteq \mathcal{F}$ follows from Lemma 7.1. This inclusion is strict since every finitely generated subvariety in the interval $\left[\mathbf{B}_{\mathbf{0}}, \mathbf{A}_{\mathbf{2}}\right]$ is nonsmall.

(ii) Suppose that $\mathbf{U}, \mathbf{V} \in \mathcal{F}$. If $\mathbf{U}, \mathbf{V} \in\left[\mathbf{B}_{\mathbf{0}}, \mathbf{A}_{\mathbf{2}}\right]$, then by referring to Figure 5 , it is easy to see that $\mathbf{U} \cap \mathbf{V} \in \mathcal{F}$. If either $\mathbf{U}$ or $\mathbf{V}$ belongs to $\mathcal{I}_{5}$, say $\mathbf{U} \in \mathcal{I}_{5}$, then $\mathbf{U} \in \mathcal{F} \cap \mathcal{I}_{5}=\mathcal{S}$ by Lemma 7.1 so that $\mathbf{U} \cap \mathbf{V} \in \mathcal{S}=\mathcal{F} \cap \mathcal{I}_{5}$. Hence, $\mathbf{U} \cap \mathbf{V} \in \mathcal{F}$ in any case. It is clear that $\mathbf{U} \vee \mathbf{V} \in \mathcal{F}$ so that $\mathcal{F}$ is a lattice. By Corollary 5.6(ii), the nonfinitely generated variety $\langle\omega, 0\rangle_{4}$ is the complete join of infinitely many finitely generated varieties. Hence, the lattice $\mathcal{F}$ is incomplete.

(iii) Since $\mathcal{S}=\mathcal{F} \cap \mathcal{I}_{5}$ by Lemma 7.1 and $\mathcal{F}$ is a lattice by part (ii), the set $\mathcal{S}$ is a lattice. The complete join $\bigvee \mathcal{S}$ contains the varieties $\mathbf{L}_{0}, \mathbf{L}_{1}, \ldots$ and so is nonsmall by Lemma 4.3. Hence, the lattice $\mathcal{S}$ is incomplete.

Proof of TheOrem B. The algorithm presented in this proof potentially involves verification of the following conditions: 
(C1) $\mathbf{B}_{\mathbf{0}} \vDash \Sigma$;

(C2) $\Sigma$ contains some diverse identity;

(C3) $\langle\omega, 0\rangle_{4} \vDash \Sigma$ and $|0\rangle_{4} \not \models \Sigma$;

(C4) $\langle 0, \omega\rangle_{4} \vDash \Sigma$ and $\left\langle\left. 0\right|_{4} \not \models \Sigma\right.$.

It follows from Proposition 6.4 and its dual result that deciding if these conditions hold are problems with complexity $\mathcal{O}(n k)$.

First suppose that (C1) does not hold. Then $\mathbf{A}_{2} \Sigma=\overline{\mathbf{B}}_{\mathbf{0}} \Sigma$ is a variety in the interval $\mathcal{I}_{5}$. By Proposition 3.3, the variety $\mathbf{A}_{\mathbf{2}} \Sigma$ is both finitely generated and small if (C2) holds, and neither finitely generated nor small otherwise.

Now suppose that (C1) holds. Then the variety $\mathbf{A}_{\mathbf{2}} \Sigma$ belongs to the interval $\left[\mathbf{B}_{\mathbf{0}}, \mathbf{A}_{\mathbf{2}}\right]$ and is nonsmall since $\mathbf{B}_{\mathbf{0}}$ is nonsmall [9]. By referring to Figure 5, it is easy to see that the nonfinitely generated subvarieties in $\left[\mathbf{B}_{\mathbf{0}}, \mathbf{A}_{\mathbf{2}}\right]$ constitute the intervals $\left[\langle\omega, 0\rangle_{4},\left\langle\left.\omega\right|_{1}\right]\right.$ and $\left[\langle 0, \omega\rangle_{4},|\omega\rangle_{1}\right]$, and that the variety $\mathbf{A}_{\mathbf{2}} \Sigma$ is nonfinitely generated if and only if either (C3) or (C4) holds.

The algorithm in this proof is summarized in the flowchart in Figure 6.

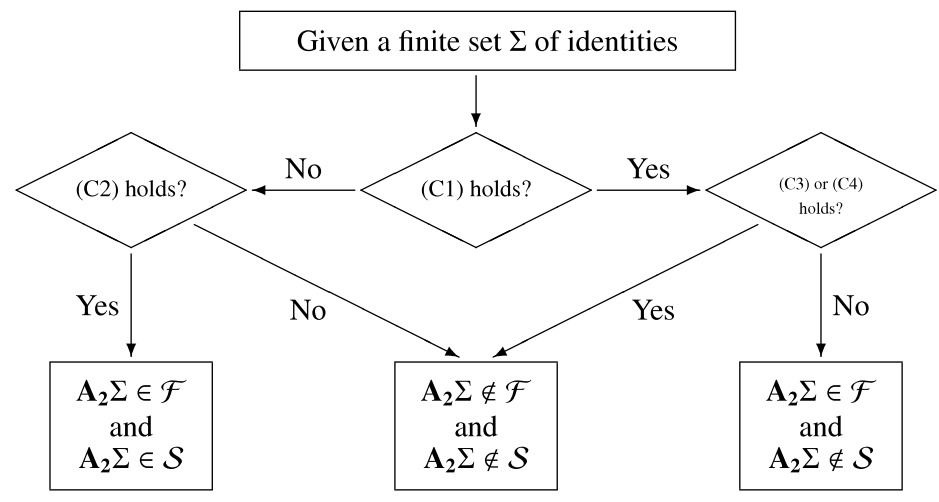

FIGURE 6. The algorithm in the proof of Theorem B.

\section{Acknowledgement}

The author is very grateful to Professor Mikhail V. Volkov for several important discussions and providing information on Lemma 6.3 and the semigroup $P$.

\section{References}

[1] J. Almeida, Finite Semigroups and Universal Algebra (World Scientific, Singapore, 1994).

[2] K. Auinger and M. B. Szendrei, 'On identity bases of epigroup varieties', J. Algebra 220 (1999), 437-448.

[3] S. Burris and H. P. Sankappanavar, A Course in Universal Algebra (Springer, New York, 1981).

[4] C. C. Edmunds, 'Varieties generated by semigroups of order four', Semigroup Forum 21 (1980), 67-81. 
[5] T. E. Hall, S. I. Kublanovsky, S. Margolis, M. V. Sapir and P. G. Trotter, 'Algorithmic problems for finite groups and finite 0-simple semigroups', J. Pure Appl. Algebra 119 (1997), 75-96.

[6] S. I. Kublanovsky, 'On the Rees-Sushkevich variety', Unpublished manuscript.

[7] S. I. Kublanovsky, E. W. H. Lee and N. R. Reilly, 'Some conditions related to the exactness of Rees-Sushkevich varieties’, Semigroup Forum 76 (2008), 87-94.

[8] E. W. H. Lee, 'Identity bases for some non-exact varieties', Semigroup Forum 68 (2004), $445-457$.

[9] - 'Subvarieties of the variety generated by the five-element Brandt semigroup', Internat. J. Algebra Comput. 16 (2006), 417-441.

[10] - 'On the complete join of permutative combinatorial Rees-Sushkevich varieties', Int. J. Algebra 1 (2007), 1-9.

[11] - 'Combinatorial Rees-Sushkevich varieties are finitely based', Internat. J. Algebra Comput. 18 (2008), 957-978.

[12] E. W. H. Lee and N. R. Reilly, 'The intersection of pseudovarieties of central simple semigroups', Semigroup Forum 73 (2006), 75-94.

[13] _ 'Centrality in Rees-Sushkevich varieties', Algebra Universalis 58 (2008), 145-180.

[14] E. W. H. Lee and M. V. Volkov, 'On the structure of the lattice of combinatorial Rees-Sushkevich varieties', in: Semigroups and Formal Languages (eds. J. M. André et al.) (World Scientific, Singapore, 2007), pp. 164-187.

[15] - 'Limit varieties generated by completely 0 -simple semigroups', Preprint.

[16] S. A. Malyshev, 'Permutational varieties of semigroups whose lattice of subvarieties is finite', in: Modern Algebra (Leningrad Univ., Leningrad, 1981), pp. 71-76 (in Russian).

[17] G. I. Mashevitzky, 'On bases of completely simple semigroup identities', Semigroup Forum 30 (1984), 67-76.

[18] - 'Varieties generated by completely 0-simple semigroups', in: Semigroups and their Homomorphisms (ed. E. S. Lyapin) (Ross. Gos. Ped. Univ., Leningrad, 1991), pp. 53-62 (in Russian).

[19] _ 'On the finite basis problem for completely 0-simple semigroup identities', Semigroup Forum 59 (1999), 197-219.

[20] D. Rees, 'On semi-groups', Proc. Cambridge Philos. Soc. 36 (1940), 387-400.

[21] N. R. Reilly, 'The interval $\left[\mathbf{B}_{2}, \mathbf{N B}_{2}\right]$ in the lattice of Rees-Sushkevich varieties', Algebra Universalis 59 (2008), 345-363.

[22] M. V. Sapir, 'On Cross semigroup varieties and related questions', Semigroup Forum 42 (1991), 345-364.

[23] A. K. Sushkevich, 'Über die endlichen Gruppen ohne das Gesetz der eindeutigen Umkehrbarkeit', Math. Ann. 99 (1928), 30-50 (in German).

[24] R. Tarjan, 'Depth-first search and linear graph algorithms', SIAM J. Comput. 1 (1972), 146-160.

[25] A. N. Trahtman, 'Identities of a five-element 0-simple semigroup', Semigroup Forum 48 (1994), 385-387.

[26] B. M. Vernikov and M. V. Volkov, 'Lattices of nilpotent semigroup varieties. II', Izv. Ural. Gos. Univ. Mat. Mekh. 1 (1998), 13-33 (in Russian).

[27] M. V. Volkov, 'The finite basis problem for finite semigroups', Sci. Math. Jpn. 53 (2001), 171-199.

[28] - 'On a question by Edmond W. H. Lee', Izv. Ural. Gos. Univ. Mat. Mekh. 7 (2005), $167-178$.

EDMOND W. H. LEE, Department of Mathematics, Simon Fraser University, Burnaby, British Columbia V5A 1S6, Canada

e-mail: ewl@sfu.ca 